\title{
Novel Ensemble Algorithm for Multiple Activity Recognition in Elderly People Exploiting Ubiquitous Sensing Devices
}

\author{
Sidrah Liaqat, Kia Dashtipour, Syed Aziz Shah, Ali Rizwan, Abdullah Alhumaidi Alotaibi, Turke Althobaiti, \\ Kamran Arshad, Khaled Assaleh, Naeem Ramzan
}

\begin{abstract}
Ambient assisted living is good way to look after ageing population that enables us to detect human's activities of daily living (ADLs) and postures, as number of older adults are increasing at rapid pace. Posture detection is used to provide the assessment for monitoring the activity of elderly people. Most of the existing approaches exploit dedicated sensing devices as as cameras, thermal sensors, accelerometer, gyroscope, magnetometer and so on. Traditional methods such as recording data using these sensors, training and testing machine learning classifiers to identify various human postures. This paper exploits data recorded using ubiquitous devices such as smart phones we use on daily basis and classify different human activities such as standing, sitting, laying, walking, walking downstairs and walking upstairs. Moreover, we have used machine learning and deep learning classifiers including random forest, KNN, logistic regression, multilayer perceptron, decision tree, QDA and SVM, convolutional neural network and long short-term memory as ground truth and proposed a novel ensemble classification algorithm to classify each human activity.

The proposed algorithm demonstrate classification accuracy of $98 \%$ that outperforms other algorithms.
\end{abstract}

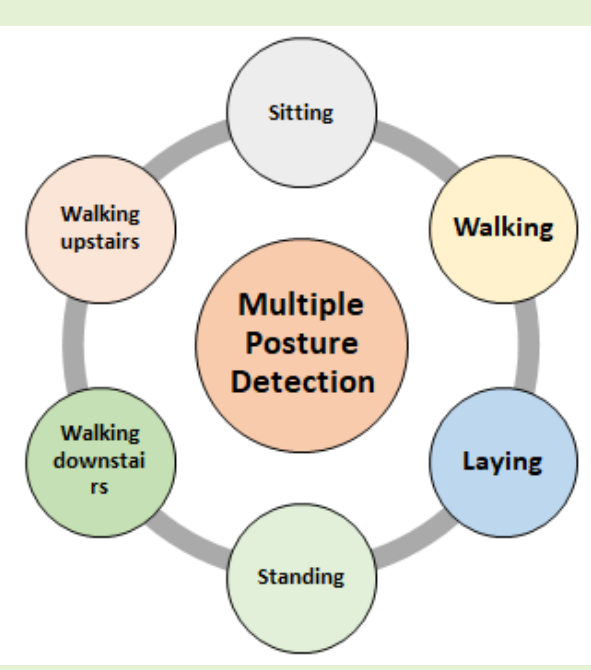

Index Terms-Posture detection, Ensemble algorithm,Deep Learning, Machine Learning, ubiquitous devices.

\section{INTRODUCTION}

Recent years have witnessed a single independent lifestyle among elderly people, worldwide. Studies indicate that the total number of elderly people (65+) will reach 1.2 billion by year 2025 [1, 2]. However, large chunk of ageing population cannot continue independent living due to mental and physical impairments [3]. In many applications, security, healthcare,

Sidrah Liaqat and Naeem Ramzan are with School of Engineering and Computing, University of the West of Scotland, Paisely PA1 2BE, UK.(E-mail: b00376760@studentmail.uws.ac.uk, Naeem.Ramzan@uws.ac.uk).

Kia Dashtipour is with School of Computing, Merchiston Campus, Edinburgh Napier University, Edinburgh EH10 5DT, Scotland, U.K. (Email: k.dashtipour@napier.ac.uk).

Syed Aziz Shah is with Research Centre for Intelligent Healthcare, Coventry University, Coventry CV15FB, UK. (E-mail: syed.shah@coventry.ac.uk ).

Ali Rizwan is with Qatar Mobility Innovations Center, Qatar, Qatar Science Technology Park, Doha, Qatar. (E-mail: arizwan@qmic.com ).

Abdullah Alhumaidi Alotaibi is with Department of Science and Technology, college of Ranyah, Taif University, P.O. Box 11099, Taif 21944, Saudi Arabia. (E-mail: a.alhumaidi@tu.edu.sa).

Turke Althobaiti is with Faculty of Science, Northern Border University, Arar 91431, Saudi Arabia. (E-mail: Turke.althobaiti@nbu.edu.sa).

Kamran Arshad and Khaled Assaleh are with College of Engineering and IT, Ajman University, Ajman, United Arab Emirates (E-mail: k.arshad@ajman.ac.ae,k.assaleh@ajman.ac.ae). indoor and outdoor monitoring, human posture and position is determined from the dedicated sensors deployed within area of interest. These include radar sensors, camera-based sensors, thermal sensors and other radio frequency based sensors. Due to limited healthcare resources, it is essential to recommend a technology that can support remote monitoring of vulnerable people so that they can live independently. In modern times humans are less active and spend more time in a sitting position which causes a fall in the strength of muscles $[4$, $5,6]$

This inactive lifestyle leads to neck, shoulder and back pain due to the wrong posture of a human. Therefore, it is important to control and detect the posture of a human. But, there is a lot of challenges such as lighting, background, human shape and self-occlusion. For posture recognition different methods are used which are sensitive to background noise. Some deep learning techniques deal with depth information and skeleton to avoid background noise and robust to viewpoint $[7,8,9]$. In this paper, we exploited the data obtained using ubiquitous devices such as smartphones used by nearly everyone and applied different machine learning algorithms to detect ADL and postures observed in daily life. These include walking in indoor setting, sitting down on chair, standing up from chair, 
laying on bed, going up on stairs, going down on chair so on. Machine learning classification algorithms used in this paper are as follows:random forest, KNN, logistic regression, multilayer perceptron, decision tree, QDA and SVM, CNN and LSTM for monitoring human posture. In addition, the novel ensemble approach is used to detect postures such as standing, sitting, laying, walking, walking downstairs and walking upstairs. It is vital to develop a novel approach to detect multiple postures as most of the current approaches are focus on the binary detection of posture, for example, most of the current studies only used to detect walking and standing. The major contribution of this study are outlined below:

- We introduce a novel low-complexity machine learning algorithm based on the ensemble classifier to detect multiple posture using smartphone data.

- Additionally, the novel ensemble approach is used to combine deep learning (CNN, LSTM) and machine learning classifiers (SVM, QDA, logistic regression, MLP etc) methods.

- A comparison of performance between existing machine learning algorithm and the proposed algorithm have been shown.

This paper is organized as follows: section II demonstrates an extensive literature review on existing techniques, limitations and research gaps, section III provides the methodology, section IV provides experimental results and discussion and finally section $\mathrm{V}$ concludes the paper.

\section{Related WORK}

This section presents a comprehensive literature review on different sensing techniques, state-of-the-art classification algorithms used to detect human postures within area of interest [10, 11].

It is worth to mention that, there are lots of studies published using machine learning and deep learning for different tasks such as natural language processing $[12,13,14,15,16,17$, $18,19,20]$, speech enhancement $[21,22,23,24,25,26,27]$, cyber-security [28], fall detection [29, 30, 31, 32, 33, 34] and etc.

However, For improving quality of life many, developing technologies are being used for a person at home or indoor. Most of these techniques are remote control, video surveillance and telehealth care. The main objective of this system is the safety of humans. For this purpose non-invasive methods are used which are based on video processing obtained from distributed cameras. In a smart automation home, the behaviour of a person is monitored and possible alarm conditions are defined. There are many motion analysis procedures and computer vision that are used to obtain the objects and events from the scene. Then classification and recognition depend on pre-defined ontology. In this case, a fallen person is an event which is predicted when the person's position is laying on bed for particular period of time. The proposed approach is divided into two steps; the first step is a classification of posture frame by frame which is visual object based classification and machine learning model creates probabilistic projection maps (PPMs) exploited by Bayesian classification algorithm.
The second step is the posture classification using tracking information i.e., temporarily integrated. The accuracy of a model is increased when state transition is added. These classification techniques are useful in the detection of alarming states that is either person is fallen or laying down for a certain period [35].

In addition to that, the United Kingdom and European elderly population is increasing at rapid pace, as a result life expectancy has also raised and dramatic drop in birth rates. The proportion of ageing population considering elderly people (65+) iex expective to rise by $17.9 \%$ in 2007 to $53.5 \%$ in 2060 [36]. Therefore, an effort is required so that elder people can live a maximum life span with a minimum support system. So, there is a universal project which detects the person falling situation and other health behaviour changes. This improves the medical involvement and security of the elder person. This is achieved by small wireless tags on some important places of the body like elbows, wrists, ankles, hips, knees and shoulders. Some tags are places on the bed and chair which is used to detect sitting and lying posture. There are few approaches to detect these activities and features are extracted from the data of the sensor. The first method is to use accelerates. This detects acceleration due to gravity and angular velocity with respect to acceleration the earth gravitational force. The second method is with gyroscopes, which give orientation along with one and multiple axis. The third approach is posture reconstruction with visual detection from still images or video. The fourth technique is based on the $3 \mathrm{D}$ position of pointers which are placed on an object like the human body. Then a selection of features which are coordinate system, location of various parts of the human body considering coordinate system. These features are then subjected to different machine learning classifier, among the SVM classifier provide best classification results considering multi-class classification problem. The activities to identify are falling, walking, sitting, lying down and the process of lying down and sitting down. With the help of idle states like sitting and lying, the health problem is detected [37, 38]. To manage the problems of the elder and disable (wheelchair users) many supportive systems have been designed to give them independent life. A smart wheelchair used by an elderly or paralyzed person enhances the capabilities with simple wheelchair with BANs, cloud computing and multi-sensors [39] [40] [41].

Existing research work conducted on these technology driven wheelchairs uses vital signals such as cardiac activity, blood pressure, glucose level, respiratory rate and son and human activities, detection of obstacle and navigation [42, 43] [44]. The wheelchair that has cushion-based technology uses pressure sensors to identify different body postures of patient under usage. This system generates an alarm if a dangerous posture is being detected by a sensor. The optimization method is applied to data of pressure sensor and then different classifiers are applied to find the best classifier for smart pressure sensor cushion wheelchair. The proposed method for the detection of posture consists of following three main steps: data acquisition classification to determine different human postures, choosing optimum sensor and its corresponding tech- 
niques and comparing results of different sensing technologies. Following classification models are used: Decision tree, Naïve Bayes, k-nearest neighbour, support vector machine and multilayer perception. Performance analysis also considered Body Mass Index for the evaluation of individual physical differences [45, 38]. The RGBD sensors (Microsoft Kinect) are used for the detection and recognition of activity. RGBD sensor is an input sensor and extraction of features depends on various human postures and movements. This algorithm is based on two-layered maximum entropy Markov model (MEMM), which consists of human activity in a set of subactivities. Human activities that are under consideration are cooking, brushing teeth, drinking water, talking on the phone and so on. For example, drinking water consists of few steps; drinking water from glass. There is a problem that every person do different task in a different rate, to overcome this onthe-fly graph structure selection is used which tunes according to the variation in speed and motion pattern of a person. This is performed by Prime sense skeleton tracking system. This method allows to obtained efficient and precise performance in identification of activities of daily living performed on regular basis [46].

Human poses detection is an increasing the trend in research community. There is a problem of joint localization in human images and videos like elbow, wrist, knee, etc. which is known as Human Pose estimation (HPE). Previously graphical models were used for pose estimation. But, the image base technique comprises two main steps; the first method is to extract features from original data and then classifiers are applied to them. Deep learning classifiers have influential abilities, therefore, a Convolutional neural network is used in video-based HPE. The deep neural network defines many computer vision problems, especially in HPE tasks. Therefore, Deep Full-Body-HPE (DFB-HPE) technique from Red-GreenBlue stationary images. There are fifteen positions of the joint which used in different application gesture recognition, human-robot interaction and sports performance analysis. The DFB-HPE detects the daily activities of a person then extracted features are fed to support vector machine classifier. The two public datasets J-HMDB and CAD-60 are used for the training of DFB-HPE. The results retrieved indicate the efficacy of exploiting the ConvNet-based posture detection to bring improvement in the activity detection levels [47].

In a smart home environment human activities (health, alarming situations and daily fitness) recognition is an important issue. Nowadays, smartphones and smartphone base applications are used everywhere. Most of the smartphones have an orientation sensor, tri-axial accelerometer and linear accelerator. This embedded sensor gives information on acceleration and rotation around $\mathrm{X}, \mathrm{Y}$ and $\mathrm{Z}$ axes. With these sensors, data is collected and then some machine learning classifiers are applied to them. This system is implemented in android smartphones. Smart phones are now vital because of easy internet availability and many mobile applications. Due to these features, phone-based detection of human activity is possible, which records daily data of activities and sends an alert message if something anomalous happens. There two types of data analysis in this approach; statistical eval- uation that comprise maximum, minimum, mean, standard deviation, kurtosis and so on that leverages on the machine learning algorithms. This approach detects simple activities like walking, running and sitting. J48 in long-term analysis and standard deviation in short-term analysis give better accuracy [48]. There is a wearable sensor which is designed for ADL detection and this sensor can wear on the wrist, thigh and ankle. This accelerometer sensors provides statistical features such as the mean of the overall acceleration recorded for a period of time [49].

In addition, the modified hierarchical support vector machine was used to detection activities of daily living such as walking up on chairs and going down on stairs, stationary position and running [50]. The wavelet transform was also used to detect walking activities using original data with the Knearest neighbour classifier [51]. Human activity recognition (HAR) attaining a lot of consideration in modern times. The human activity recognition exploiting ubiquitious devices such as embedded smartphone sensors is a traditional time-series data classification research challenge and open area that need to be address [52]. There is a conventional neural network classifier that is used for feature extraction and HAR using smartphone sensors. Features are automatically extracted using a multi-level convolutional network with alternating convolution and pooling layers. This approach gives better accuracy as compared to the existing techniques. This high accuracy is for the moving activities which are walking upstairs and downstairs [53]. The posture detection of humans is used in many applications like indoor/outdoor monitoring, surveillance, virtual reality to moving pictures and amusement. For disable and elder people assistance, video-based monitoring is used which detect their behaviour and posture. The system is aimed to fulfil real-time constraints and process several frames and then apply machine learning for the posture (walking, lying down, and sitting) detection using extracted features. There are three tests:

- The effectiveness in detection of posture on the same videos from where the training phase has been given.

- When other videos are taken from the visual sensor (camera).

- When videos are recorded from various camera-based sensors for training sets [54].

In recent times for a military application, surveillance and assisted living HAR is important to research. There are various sensors like GPS, radars, LIDAR and cameras which are used for these applications. Among all Doppler radar is emerging due to motion analysis and it works through building walls, foliage and clothes. Also, its low power and cheap components increase its popularity outdoor and on a large scale. For these applications, it is important to know whether the objective is alone or in a group of people and the rough location of the target. This approach is divided into subject classification, people counting, human activity classification and rough localisation using a micro-Doppler radar system. The two-directional two-dimensional principal component analysis (2D2PCA) is exloit to generate time-domain statistical features to train the machine learning classifiers. This improves the 
result of KNN and SVM classifiers. Also, CNN is used which gives a better result as compare to SVM and KNN [55].

In addition, a real-time visual sensor-based (exploiting cameras) surveillance methods can reliably detection, track and identify a person moving within area of interest. For the recognition of moving things background subtraction, flow examination, temporal-differential method and dynamic threshold methods are used. Finally, propose system uses the Gaussian function and MATLAB. There are two kinds of activities normal and abnormal which are classified with the help of SVM. This model generates an alarm if abnormal activity is detected [56]. Modern medical science has proven that the behaviour of humans affects human health. These days people are doing work in the same posture for a long time even their bodies give alarms of tiredness. Therefore people need a reminder to either change the posture or do some rest. For smart city development not only living and transportation supervised also health must smartly supervise. With the introduction of AI and big data analysis, researchers and academics are finding the relationship between health and human daily activities. Human activity detection and identification can be applied in various application, specifically for patient monitoring [57]. Continuous monitoring of human postures should be detected irrespectable of geometrical locations and age group involved. For this requirement, LoRa(Long range) multi-sensor based system is proposed. LoRa is low power and extra-long transmission of the signal [58].

This sensing system exploits Inertial Measurement Uni IMU which is equipped with an Arduino Mega board, and a LoRa shield. These sensors have an accelerometer and magnetometer which receive data and transmit to Arduino board which is then connected with LoRa shield and transfer data to LoRa gateway and server. This clothing records daily activities like running, walking, going downstairs or upstairs and standing. These machine learning time-domain statistical features are extracted from the data set and then fed to different classifiers [59]. Data acquirement methods are Bluetooth, internet and wi-fi but they are limited due to their small range. The posture detection using LoRa comprises of four modules; sensor gives information of posture, wireless communication, recognition of human posture and user interface. Targeting at LoRa's low transmission power and transmission frequency for small duration of time, multiprocessing techniques is utilized that include de-noising of data, expanding of data using window slider, extraction of features and finally, Random forest classifier is trained with selected features. Therefore, the proposed approach for long term detection of human posture consist of multi-sensors and LoRa [60]. Finally, Kulikajevas [61] proposed a novel deep learning model based on the MobileNetV2 for remove the current problem of posture detection related to visibility of human such as occlusion. The approach received accuracy of $91.47 \%$. In addition, Dai et al. [62] Introduced deep learning approach which estimate pedestrian detection using ResNet-50. The performance of the approach hardly achieved $80 \%$.
TABLE I

MACHINE LEARNING METHODS WITH THEIR PARAMETERS

\begin{tabular}{|l|l|l|}
\hline Method & Parameters & Training time \\
\hline KNN & Euclidean distance & $5 \mathrm{~m} \mathrm{22} \mathrm{s}$ \\
\hline SVM & RBF Kernal & $5 \mathrm{~m} \mathrm{36} \mathrm{s}$ \\
\hline Random Forest & Max depth $=3$ & $5 \mathrm{~m} 22 \mathrm{~s}$ \\
\hline KNN & 5 & $4 \mathrm{~m} 21 \mathrm{~s}$ \\
\hline Logistic regression & Penalty $=12$ & $4 \mathrm{~m} 6 \mathrm{~s}$ \\
\hline QDA & tol $=0.0001$ & $8 \mathrm{~m} \mathrm{36} \mathrm{s}$ \\
\hline LSTM & 2-layered & $16 \mathrm{~m} 49 \mathrm{~s}$ \\
\hline CNN & $\begin{array}{l}\text { dropout }=0.2 \\
10 \text { layers }\end{array}$ & $10 \mathrm{~m} 12 \mathrm{~s}$ \\
\hline
\end{tabular}

\section{Methodology}

In this section, we discuss the novel technique to identify different human postures. The Fig displays the proposed framework for posture detection.

\section{A. Time-domain Statistical Feature Extraction}

Different time-domain statistical features to train the machine learning classifiers such as minimum, maximum, percentile and average are used. The value of each time-domain statistical features is extracted exploiting single window size. For instance, a 90 seconds windows size was used when extracted time-domain statistical features considering the minimum, maximum, percentile and average are calculated.

\section{B. Machine Learning Classification Techniques}

After the extracting the feature to train the classifier, we have used classification algorithms as discussed. In addition, the novel ensemble approach including, bagging, blending and stacking used to predict different postures. In order to train the aforementioned classifiers, there are a set of parameters used to train the classification algorithm for evaluation purpose. The scikit-learn was used to training, test and validated the classifiers and keras with tensor-flow background is used to train the deep learning classifiers. Table I displays the parameters used to train the classifiers.

\section{Deep Learning Classification Algorithms}

In this section, we discuss the proposed CNN and LSTM architecture.

CNN: The convolutional neural network framework has been inspired from [63] [64] [65] and it has input, hidden and output layers. The hidden has been made up from convolutional, max pooling and fully connected layers. In this experiment, we used 10-layered CNN layers as shown in Table II.

LSTM: The LSTM has used a similar framework which is used in [31] [66] [21] contains input, two stacked Long short-term memory and one output layer. The Long short-term memory possess two bidirectional Long short-term memory layers having 64 along associated 64 cells, and corresponding dropout layer having an overall probability value of 0.2 , an individual dense LSTM layer having two neurons and one softmax activation layer. 


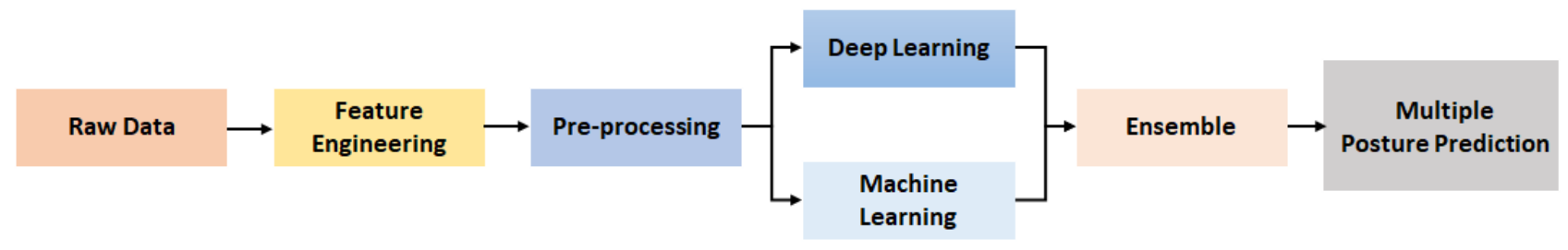

Fig. 1. Proposed Framework multiple posture detection

TABLE II

CNN Architecture (Conv - convolutional layer, MaxPool - Maxpooling layer, Globalmaxpool - Global Max Pooling layer, FC - Fully CONNECTEd LAYER, RELU - RECTIFIEd LineAR UNit ActiVATION

\begin{tabular}{|c|c|c|c|c|c|c|c|c|c|c|}
\hline Layer & 1 & 2 & 3 & 4 & 5 & 6 & 7 & 8 & 9 & 10 \\
\hline Type & Conv & Max & Conv & Max & Conv & Max & Conv & Global & Fc & Fc \\
\hline Filters & 16 & Pool & 32 & Pool & 64 & & 128 & Max & & \\
\hline Kernal Size & 3 & 2 & 3 & 2 & 3 & 2 & 3 & Pool & & \\
\hline Neurons & & & & & & & & & 128 & 2 \\
\hline Activation & ReLU & & ReLU & & ReLU & & ReLU & & ReLU & SoftMax \\
\hline
\end{tabular}

\section{Ensemble Models}

In this study, we used three types of ensemble classification approach, stacking, bagging and blending.

Stacking: The ensemble method combines machine learning, deep learning classification, via a meta-model are combined. The proposed model is trained on the whole dataset, then the meta-model is used on features returned from the based model. However, the based models in stacking are different. The meta-model is used to find the best models from the base model to perform the best accuracy.

Blending: It is similar to stacking, however, it is split the dataset into training, testing and validation to predict posture detection.

Bagging: The bagging is based on the subset of the dataset with replacement to make a size of the bag the same as the whole dataset. The final output formed after the combination of the output of all models. Fig 2 displays the ensemble classification approach posture detection.

\section{EXPERIMENTAL Results}

This section section discusses the results obtained using existing classification algorithms and compared its results with novel ensemble classification algorithm.

In this section we discuss the experimental results, to evaluate the performance of the approach, the machine learning classifiers such as logistic regression, $\mathrm{KNN}$, random forest, MLP, SVM, QDA and deep learning classifiers such as CNN and LSTM are trained. There are different features such as skew, minimum, maximum, average percentile are used.

Multi-Posture Detection: The multi-posture detection is based on 30 volunteers with age range from 19 to 48 . Each person performed six various daily routine activities when the participants were carrying smartphones. The data has been recorded to manually label the data. The ubiquitious devices such as smartphones data set was split into two sections, where $70 \%$ of participants data were for training and rest $30 \%$ for
TABLE III

SUMMARY OF RESULTS FOR LOGISTIC REGRESSION

\begin{tabular}{|l|l|l|l|l|}
\hline Posture & Precision & Recall & F1-score & Accuracy \\
\hline Laying & 0.98 & 0.98 & 0.98 & 98.73 \\
\hline Sitting & 0.93 & 0.93 & 0.93 & 93.11 \\
\hline Standing & 0.94 & 0.93 & 0.94 & 94.39 \\
\hline Walking & 0.96 & 0.93 & 0.94 & 96.42 \\
\hline Walking_downstairs & 0.93 & 0.95 & 0.94 & 93.23 \\
\hline Walking_upstairs & 0.9 & 0.92 & 0.91 & 91.23 \\
\hline Overall & $\mathbf{0 . 9 4}$ & $\mathbf{0 . 9 4}$ & $\mathbf{9 4}$ & $\mathbf{9 4 . 3 4 \%}$ \\
\hline
\end{tabular}

testing purpose [67]. Various performance metrics such as accuracy, precision, recall and f-measure were used to evaluate the performance of the classifiers.

$$
\begin{gathered}
\text { Precision }=\frac{T P}{T P+F P} \\
\text { Recall }=\frac{T P}{T P+F N} \\
F \_ \text {measure }=2 * \frac{\text { Precision } * \text { Recall }}{\text { Precision }+ \text { Recall }} \\
\text { Accuracy }=\frac{T P+T N}{T P+T N+F P+F N}
\end{gathered}
$$

Table III summarizes the results obtained for logistic regression classification algorithm. The particular human activity, i.e.e when the participant was laying was achieved with high classification accuracy.

Table IV summarizes the results obtained for random forest classification algorithm. In this case, the walking activity was classified with least misclassification as compared to other activities.

Table V summarizes the results obtained for K-nearest neighbour algorithm. When person was walking up on stairs, the algorithm best classification this particular activity as compared to rest of the activities performed. 


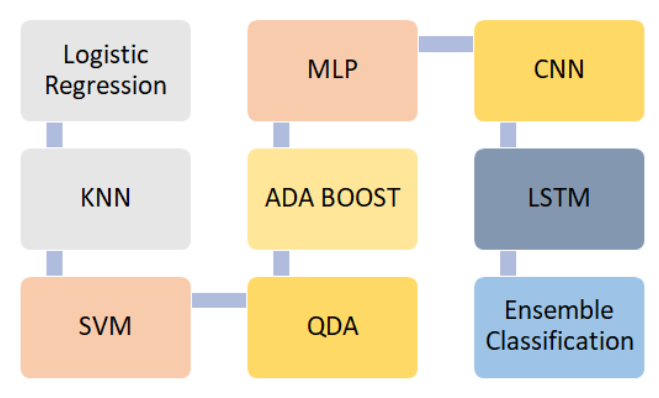

Fig. 2. Ensemble Classification Approach Posture Detection

TABLE IV

SUMMARY OF RESULTS FOR RANDOM FOREST

\begin{tabular}{|l|l|l|l|l|}
\hline Posture & Precision & Recall & F1-score & Accuracy \\
\hline Laying & 0.92 & 0.91 & 0.92 & 92 \\
\hline Sitting & 0.92 & 0.89 & 0.9 & 91.61 \\
\hline Standing & 0.9 & 0.93 & 0.91 & 90.67 \\
\hline Walking & 0.95 & 0.92 & 0.94 & 95.47 \\
\hline Walking_downstairs & 0.92 & 0.91 & 0.92 & 92.19 \\
\hline Walking_upstairs & 0.89 & 0.93 & 0.91 & 89.69 \\
\hline Overall & $\mathbf{0 . 9 5}$ & $\mathbf{0 . 9 4}$ & $\mathbf{0 . 9 5}$ & $\mathbf{9 5 . 4 3}$ \\
\hline
\end{tabular}

TABLE V

SUMMARY OF RESULTS FOR KNN

\begin{tabular}{|l|l|l|l|l|}
\hline Posture & Precision & Recall & F1-score & Accuracy \\
\hline Laying & 0.79 & 0.78 & 0.79 & 79.53 \\
\hline Sitting & 0.87 & 0.78 & 0.83 & 87.67 \\
\hline Standing & 0.82 & 0.9 & 0.86 & 82.67 \\
\hline Walking & 0.91 & 0.95 & 0.93 & 91.59 \\
\hline Walking_downstairs & 0.89 & 0.92 & 0.9 & 89.47 \\
\hline Walking_upstairs & 0.94 & 0.88 & 0.91 & 94.5 \\
\hline Overall & $\mathbf{0 . 9 7}$ & $\mathbf{0 . 9 6}$ & $\mathbf{0 . 9 7}$ & $\mathbf{9 7 . 5 8}$ \\
\hline
\end{tabular}

Table VI shows the summary of the results for SVM. As the experimental results shows the laying posture achieved better performance as compared to other postures.

Table VII show the summary of the results for Decision Tree. As the experimental results show the standing posture achieved better performance as compared to other postures.

Table VIII show the summary of the results for MLP. As the experimental results show the walking-downstairs posture achieved better performance as compared to other postures.

Table IX show the summary of the results for the Ada Boost Classifier. As the experimental results show the walking-
TABLE VII

SUMMARY OF RESULTS FOR DECISION TREE

\begin{tabular}{|l|l|l|l|l|}
\hline Posture & Precision & Recall & F1-score & Accuracy \\
\hline Laying & 0.75 & 0.74 & 0.75 & 75.49 \\
\hline Sitting & 0.86 & 0.86 & 0.86 & 86.91 \\
\hline Standing & 0.87 & 0.87 & 0.87 & 87.16 \\
\hline Walking & 0.84 & 0.83 & 0.84 & 84.37 \\
\hline Walking_downstairs & 0.87 & 0.83 & 0.85 & 87.03 \\
\hline Walking_upstairs & 0.77 & 0.81 & 0.79 & 77.67 \\
\hline Overall & $\mathbf{0 . 8 7}$ & $\mathbf{0 . 8 7}$ & $\mathbf{0 . 8 7}$ & $\mathbf{8 7 . 2}$ \\
\hline
\end{tabular}

TABLE VIII

SUMMARY OF RESULTS FOR MLP

\begin{tabular}{|l|l|l|l|l|}
\hline Posture & Precision & Recall & F1-score & Accuracy \\
\hline Laying & 0.94 & 0.93 & 0.94 & 94.51 \\
\hline Sitting & 0.92 & 0.94 & 0.93 & 92.94 \\
\hline Standing & 0.95 & 0.93 & 0.94 & 95.76 \\
\hline Walking & 0.96 & 0.97 & 0.97 & 96.38 \\
\hline Walking_downstairs & 0.98 & 0.96 & 0.97 & 98.12 \\
\hline Walking_upstairs & 0.93 & 0.95 & 0.94 & 93.97 \\
\hline Overall & $\mathbf{0 . 9 6}$ & $\mathbf{0 . 9 5}$ & $\mathbf{0 . 9 6}$ & $\mathbf{9 6 . 8 6}$ \\
\hline
\end{tabular}

downstairs posture achieved better performance as compared to other postures.

Table X show the summary of the results for the Quadratic Discriminant Analysis Classifier. As the experimental results show the walking posture achieved better performance as compared to other postures.

Table X show the summary of the results for the CNN Classifier. As the experimental results show the standing posture achieved better performance as compared to other postures.

Table XII show the summary of the results for the LSTM
TABLE VI

SUMMARY OF RESULTS FOR SVM

\begin{tabular}{|l|l|l|l|l|}
\hline Posture & Precision & Recall & F1-score & Accuracy \\
\hline Laying & 0.96 & 0.39 & 0.55 & 96.48 \\
\hline Sitting & 0.74 & 0.73 & 0.74 & 74.53 \\
\hline Standing & 0.75 & 0.74 & 0.75 & 75.49 \\
\hline Walking & 0.92 & 0.65 & 0.76 & 92.6 \\
\hline Walking_downstairs & 0.69 & 0.79 & 0.74 & 69.57 \\
\hline Walking_upstairs & 0.67 & 0.84 & 0.74 & 67.58 \\
\hline Overall & $\mathbf{0 . 6 7}$ & $\mathbf{0 . 6 6}$ & $\mathbf{0 . 6 7}$ & $\mathbf{6 7 . 5 1}$ \\
\hline
\end{tabular}

TABLE IX

SUMmARY OF RESULTS FOR AdA BOOST CLASSIFIER

\begin{tabular}{|l|l|l|l|l|}
\hline Posture & Precision & Recall & F1-score & Accuracy \\
\hline Laying & 0.65 & 0.64 & 0.65 & 65.7 \\
\hline Sitting & 0.6 & 0.59 & 0.6 & 61.94 \\
\hline Standing & 0.52 & 0.51 & 0.52 & 52.64 \\
\hline Walking & 0.62 & 0.61 & 0.62 & 62.32 \\
\hline Walking_downstairs & 0.68 & 0.67 & 0.68 & 68.45 \\
\hline Walking_upstairs & 61 & 0.61 & 0.61 & 61.59 \\
\hline Overall & $\mathbf{0 . 6 4}$ & $\mathbf{0 . 6 3}$ & $\mathbf{0 . 6 4}$ & $\mathbf{6 4 . 4 8}$ \\
\hline
\end{tabular}


TABLE X

SUMMARY OF RESULTS FOR QUADRATIC DISCRIMINANT ANALYSIS CLASSIFIER

\begin{tabular}{|l|l|l|l|l|}
\hline Posture & Precision & Recall & F1-score & Accuracy \\
\hline Laying & 0.98 & 0.97 & 0.98 & \\
\hline Sitting & 0.7 & 0.68 & 0.69 & 70.67 \\
\hline Standing & 0.74 & 0.71 & 0.72 & 74.95 \\
\hline Walking & 0.92 & 0.47 & 0.62 & 92.4 \\
\hline Walking_downstairs & 0.59 & 0.98 & 0.74 & 59.67 \\
\hline Walking_upstairs & 0.75 & 0.81 & 0.78 & 75.89 \\
\hline Overall & $\mathbf{0 . 7 6}$ & $\mathbf{0 . 7 5}$ & $\mathbf{0 . 7 6}$ & $\mathbf{7 6 . 4 8}$ \\
\hline
\end{tabular}

TABLE XI

SUMMARY OF RESULTS FOR CNN CLASSIFIER

\begin{tabular}{|l|l|l|l|l|}
\hline Posture & Precision & Recall & F1-score & Accuracy \\
\hline Laying & 0.93 & 0.92 & 0.93 & 93.58 \\
\hline Sitting & 0.94 & 0.94 & 0.94 & 94.78 \\
\hline Standing & 0.97 & 0.96 & 0.97 & 97.91 \\
\hline Walking & 0.96 & 0.95 & 0.96 & 96.43 \\
\hline Walking_downstairs & 0.92 & 0.91 & 0.92 & 92.47 \\
\hline Walking_upstairs & 0.91 & 0.9 & 0.91 & 91.79 \\
\hline Overall & $\mathbf{0 . 9 8}$ & $\mathbf{0 . 9 8}$ & $\mathbf{0 . 9 8}$ & $\mathbf{9 8 . 5 4}$ \\
\hline
\end{tabular}

Classifier. As the experimental results show the laying posture achieved better performance as compared to other postures.

Table XII shows the summary of the results for the Ensemble Stacking method. As the experimental results show the laying posture achieved better performance as compared to other postures. The overall training accuracy was $98.91 \%$.

Table XIV show the summary of the results for the Ensemble blending method. As the experimental results show the laying posture achieved better performance as compared to other postures. The overall training accuracy was $99.61 \%$. It is worth to mention that, the overall true positive is 953 , true negative 959, and false positive is 41 and false negative is 43 .

Table XIV shows the summary of the results for the Ensemble bagging method. As the experimental results show the standing posture provide best classification accuracy when its performance were compared with other human postures.

TABLE XII

SUMMARY OF RESULTS FOR LSTM CLASSIFIER

\begin{tabular}{|l|l|l|l|l|}
\hline Posture & Precision & Recall & F1-score & Accuracy \\
\hline Laying & 0.95 & 0.94 & 0.95 & 95.46 \\
\hline Sitting & 0.94 & 0.94 & 0.94 & 94.1 \\
\hline Standing & 0.89 & 0.89 & 0.89 & 89.45 \\
\hline Walking & 0.95 & 0.95 & 0.95 & 95.7 \\
\hline Walking_downstairs & 0.94 & 0.93 & 0.94 & 94.01 \\
\hline Walking_upstairs & 0.92 & 0.91 & 0.92 & 92.18 \\
\hline Overall & $\mathbf{0 . 9 5}$ & $\mathbf{0 . 9 5}$ & $\mathbf{0 . 9 5}$ & $\mathbf{9 5 . 1 2}$ \\
\hline
\end{tabular}

TABLE XIII

Summary of REsults for ENSEMBLE StAcking METHOd

\begin{tabular}{|l|l|l|l|l|}
\hline Posture & Precision & Recall & F1-score & Accuracy \\
\hline Laying & 0.96 & 0.95 & 0.96 & 96.59 \\
\hline Sitting & 0.90 & 0.91 & 0.91 & 91.97 \\
\hline Standing & 0.94 & 0.93 & 0.94 & 94.66 \\
\hline Walking & 0.93 & 0.92 & 0.93 & 93.94 \\
\hline Walking_downstairs & 0.91 & 0.90 & 0.91 & 91.63 \\
\hline Walking_upstairs & 0.92 & 0.91 & 0.92 & 92.81 \\
\hline Overall & $\mathbf{0 . 9 4}$ & $\mathbf{0 . 9 3}$ & $\mathbf{0 . 9 4}$ & $\mathbf{9 4 . 8 2}$ \\
\hline
\end{tabular}

TABLE XIV

SUMMARY OF RESULTS FOR ENSEMBLE BLENDING METHOD

\begin{tabular}{|l|l|l|l|l|}
\hline Posture & Precision & Recall & F1-score & Accuracy \\
\hline Laying & 0.98 & 0.97 & 0.98 & 98.69 \\
\hline Sitting & 0.97 & 0.96 & 0.97 & 97.51 \\
\hline Standing & 0.96 & 0.95 & 0.96 & 96.47 \\
\hline Walking & 0.95 & 0.94 & 0.95 & 95.49 \\
\hline Walking_downstairs & 0.94 & 0.93 & 0.94 & 94.69 \\
\hline Walking_upstairs & 0.93 & 0.92 & 0.93 & 93.67 \\
\hline Overall & $\mathbf{0 . 9 6}$ & $\mathbf{0 . 9 5}$ & $\mathbf{0 . 9 6}$ & $\mathbf{9 6 . 5 4}$ \\
\hline
\end{tabular}

TABLE XV

SUMmARY OF RESULTS FOR ENSEMBLE BAGgING METHOD

\begin{tabular}{|l|l|l|l|l|}
\hline Posture & Precision & Recall & F1-score & Accuracy \\
\hline Laying & 0.96 & 0.95 & 0.96 & 96.6 \\
\hline Sitting & 0.94 & 0.93 & 0.94 & 93.98 \\
\hline Standing & 0.96 & 0.95 & 0.96 & 96.93 \\
\hline Walking & 0.95 & 0.94 & 0.95 & 95.87 \\
\hline Walking_downstairs & 0.92 & 0.91 & 0.92 & 92.97 \\
\hline Walking_upstairs & 0.9 & 0.9 & 0.9 & 90.09 \\
\hline Overall & $\mathbf{0 . 9 3}$ & $\mathbf{0 . 9 2}$ & $\mathbf{0 . 9 3}$ & $\mathbf{9 3 . 7 5}$ \\
\hline
\end{tabular}

The overall training accuracy was $98.99 \%$.

\section{A. Discussion}

The human posture detection is important area of research because there are more sectors such as healthcare will gain use of technology. The home healthcare use different technologies received more attention to improve the lives of people who required special care. Therefore, its important to develop a novel approach to remotely control the elderly people.

In order to train the classification algorithms, the scikitlearn and keras with tensor flow background are used to train the model. It is to be noted that the deep learning classifiers are taken more time to train as compared to machine learning classifiers. However, the ensemble classification achieved better performance as compared to machine learning and deep learning classifiers.

The deep learning model take long time to train and also they are black-box. Therefore, we cannot find out if the prediction for the posture are correct.

Table XVI indicates the comparison of different classification techniques and comparison with proposed novel algorithm. The results clearly indicate that the proposed novel ensemble algorithm outperformed other classifications algorithms when results were evaluated in terms of aforementioned performance metrics. Ronao et al. [53] developed human activity detection using smart-phones, it is worth to mention that the approach is only used CNN model. In addition, Jiang et al. [68] developed approach using wearable sensors based on the deep CNN. It is to be noted that, the aforementioned studies, their data set is publicly available online.

\section{CONCLUSION}

It is important to develop a novel approach to independently control and monitor to elderly and vulnerable without help from other people. Therefore, in this paper, we developed novel ensemble algorithms based on multiple postures such 
TABLE XVI

COMPARISON WITH STATE-OF-THE-ART APPROACHES

\begin{tabular}{|l|l|l|l|l|}
\hline Ref & Precision & Recall & F-measure & Accuracy \\
\hline Ronao et al. [53] & 0.95 & 0.95 & 0.95 & 95.43 \\
\hline Wong et al. [69] & 0.81 & 0.80 & 0.81 & 81.58 \\
\hline Jiang et al. [68] & 0.96 & 0.96 & 0.96 & 96.23 \\
\hline Hassan et al.[70] & 0.94 & 0.93 & 0.94 & 94.67 \\
\hline Murad et al.[71] & 089 & 0.89 & 0.89 & 90.07 \\
\hline Our Approach & 0.98 & 0.97 & 0.98 & 98.23 \\
\hline
\end{tabular}

as sitting, standing, walking, walking upstairs, walking downstairs and laying. To examine the overall the performance of the proposed algorithm, the machine learning and deep learning classifiers are trained and additionally, the novel ensemble approach based on deep learning and machine learning classifiers are used and the novel ensemble approach is used to improve the performance of the approach. The initial experimental results indicate that the proposed ensemble algorithm provide best classification performance when compared to other existing algorithms. As part of our future research, we intend to develop a novel hybrid feature selection technique based on a genetic algorithm to further enhance the performance of the approach.

\section{ACKNOWLEDGEMENT}

The work is supported in parts by EPSRC grant no $\mathrm{EP} / \mathrm{R} 511705 / 1$. In addition, this work is also supported in part by the Ajman University Internal Research Grant. The authors would like to thank the Taif University for funding this work through Taif University Research Supporting, Project number. (TURSP-2020/277), Taif University, Taif, Saudi Arabia

\section{RefERENCES}

[1] Syed Aziz Shah et al. "Sensor Fusion for Identification of Freezing of Gait Episodes Using Wi-Fi and Radar Imaging”. In: IEEE Sensors Journal 20.23 (2020), pp. 14410-14422. DOI: 10 . 1109 / JSEN . 2020 . 3004767.

[2] Syed Aziz Shah et al. "Privacy-preserving non-wearable occupancy monitoring system exploiting Wi-Fi imaging for next-generation body centric communication". In: Micromachines 11.4 (2020), p. 379.

[3] Syed Aziz Shah et al. "Privacy-Preserving Wandering Behavior Sensing in Dementia Patients Using Modified Logistic and Dynamic Newton Leipnik Maps". In: IEEE Sensors Journal 21.3 (2020), pp. 3669-3679.

[4] Jaehyun Lee et al. "Automatic Classification of Squat Posture Using Inertial Sensors: Deep Learning Approach". In: Sensors 20.2 (2020), p. 361.

[5] Syed Aziz Shah, Xiaodong Yang, and Qammer H Abbasi. "Cognitive health care system and its application in pill-rolling assessment". In: International Journal of Numerical Modelling: Electronic Networks, Devices and Fields 32.6 (2019), e2632.

[6] Fengling Jiang, Kia Dashtipour, and Amir Hussain. "A survey on deep learning for the routing layer of computer network". In: 2019 UK/China Emerging Technologies (UCET). IEEE. 2019, pp. 1-4.
[7] Alexander Toshev and Christian Szegedy. "Deeppose: Human pose estimation via deep neural networks". In: Proceedings of the IEEE conference on computer vision and pattern recognition. 2014, pp. 1653-1660.

[8] William Taylor et al. "A Review of the State of the Art in Non-Contact Sensing for COVID-19". In: Sensors 20.19 (2020), p. 5665.

[9] Yan-Ying Li et al. "Sleep posture classification with multi-stream CNN using vertical distance map". In: 2018 International Workshop on Advanced Image Technology (IWAIT). IEEE. 2018, pp. 1-4.

[10] Xiaodong Yang et al. "Contactless Finger Tapping Detection at C Band". In: IEEE Sensors Journal (2020).

[11] Xiaodong Yang et al. "5G-based user-centric sensing at C-band". In: IEEE Transactions on Industrial Informatics 15.5 (2019), pp. 3040-3047.

[12] Kia Dashtipour et al. "A novel context-aware multimodal framework for persian sentiment analysis”. In: arXiv preprint arXiv:2103.02636 (2021).

[13] Rami Ahmed et al. "Offline Arabic Handwriting Recognition Using Deep Machine Learning: A Review of Recent Advances". In: International Conference on Brain Inspired Cognitive Systems. Springer. 2019, pp. 457468.

[14] Rami Ahmed et al. "Deep neural network-based contextual recognition of arabic handwritten scripts". In: Entropy 23.3 (2021), p. 340.

[15] Kia Dashtipour et al. "Public Perception towards fifth generation of cellular networks (5G) on social media". In: Frontiers in Big Data (2021).

[16] Kia Dashtipour et al. "Sentiment Analysis of Persian Movie Reviews Using Deep Learning”. In: Entropy 23.5 (2021), p. 596.

[17] Amir Hussain et al. "Artificial Intelligence-Enabled Analysis of Public Attitudes on Facebook and Twitter Toward COVID-19 Vaccines in the United Kingdom and the United States: Observational Study". In: Journal of medical Internet research 23.4 (2021), e26627.

[18] Kia Dashtipour et al. "Persian named entity recognition”. In: 2017 IEEE 16th International Conference on Cognitive Informatics \& Cognitive Computing (ICCI* CC). IEEE. 2017, pp. 79-83.

[19] Imane Guellil et al. "A Semi-supervised Approach for Sentiment Analysis of Arab (ic+ izi) Messages: Application to the Algerian Dialect". In: SN Computer Science 2.2 (2021), pp. 1-18.

[20] Kia Dashtipour et al. "A comparative study of persian sentiment analysis based on different feature combinations". In: International Conference in Communications, Signal Processing, and Systems. Springer. 2017, pp. 2288-2294.

[21] Ahsan Adeel, Mandar Gogate, and Amir Hussain. "Contextual deep learning-based audio-visual switching for speech enhancement in real-world environments". In: Information Fusion 59 (2020), pp. 163-170.

[22] Mandar Gogate et al. "CochleaNet: A robust languageindependent audio-visual model for real-time speech en- 
hancement”. In: Information Fusion 63 (2020), pp. 273 285.

[23] Mandar Gogate, Kia Dashtipour, and Amir Hussain. "Visual Speech In Real Noisy Environments (VISION): A Novel Benchmark Dataset and Deep Learning-based Baseline System”. In: Proc. Interspeech 2020 (2020), pp. 4521-4525.

[24] Mandar Gogate et al. "Deep Neural Network Driven Binaural Audio Visual Speech Separation”. In: 2020 International Joint Conference on Neural Networks (IJCNN). IEEE. 2020, pp. 1-7.

[25] Mandar Gogate et al. "Av speech enhancement challenge using a real noisy corpus". In: arXiv preprint arXiv:1910.00424 (2019).

[26] Shibli Nisar et al. "Cognitively inspired feature extraction and speech recognition for automated hearing loss testing". In: Cognitive Computation 11.4 (2019), pp. 489-502.

[27] Alexander RT Gepperth, Thomas Hecht, and Mandar Gogate. "A generative learning approach to sensor fusion and change detection". In: Cognitive Computation 8.5 (2016), pp. 806-817.

[28] Cosimo Ieracitano et al. "Statistical analysis driven optimized deep learning system for intrusion detection". In: International Conference on Brain Inspired Cognitive Systems. Springer. 2018, pp. 759-769.

[29] Sidrah Liaqat et al. "A hybrid posture detection framework: Integrating machine learning and deep neural networks". In: IEEE Sensors Journal 21.7 (2021), pp. 9515-9522.

[30] Sidrah Liaqat et al. "A Review and Comparison of the State-of-the-Art Techniques for Atrial Fibrillation Detection and Skin Hydration". In: Frontiers in Communications and Networks (2021).

[31] Metin Ozturk et al. "A novel deep learning driven, low-cost mobility prediction approach for $5 \mathrm{G}$ cellular networks: The case of the Control/Data Separation Architecture (CDSA)". In: Neurocomputing 358 (2019), pp. 479-489.

[32] Sagar Suresh Kumar et al. "A review on wearable and contactless sensing for COVID-19 with policy challenges". In: Frontiers in Communications and Networks (2021).

[33] Zheqi Yu et al. "Energy and performance trade-off optimization in heterogeneous computing via reinforcement learning". In: Electronics 9.11 (2020), p. 1812.

[34] Andrew Churcher et al. "An Experimental Analysis of Attack Classification Using Machine Learning in IoT Networks". In: Sensors 21.2 (2021), p. 446.

[35] Rita Cucchiara et al. "Probabilistic posture classification for human-behavior analysis". In: IEEE Transactions on systems, man, and cybernetics-Part A: Systems and Humans 35.1 (2004), pp. 42-54.

[36] Donna Livesey. "Measuring the environmental goods and services sector". In: Economic \& Labour Market Review 4.12 (2010), pp. 45-58.
[37] Mitja Luštrek and Boštjan Kaluža. "Fall detection and activity recognition with machine learning". In: Informatica 33.2 (2009).

[38] Xiaodong Yang et al. "Detection of essential tremor at the $s$-band". In: IEEE journal of translational engineering in health and medicine 6 (2018), pp. 1-7.

[39] Giancarlo Fortino et al. "Cloud-assisted body area networks: state-of-the-art and future challenges". In: Wireless Networks 20.7 (2014), pp. 1925-1938.

[40] Raffaele Gravina et al. "Cloud-based Activity-aaService cyber-physical framework for human activity monitoring in mobility". In: Future Generation Computer Systems 75 (2017), pp. 158-171.

[41] Giancarlo Fortino et al. "Platform-independent development of collaborative wireless body sensor network applications: SPINE2”. In: 2009 IEEE International Conference on Systems, Man and Cybernetics. IEEE. 2009, pp. 3144-3150.

[42] Hsi-Chiang Chou, Yi-Ming Wang, and Huai-Yuan Chang. "Design intelligent wheelchair with ECG measurement and wireless transmission function". In: Technology and Health Care 24.s1 (2016), S345-S355.

[43] Syed Aziz Shah et al. "Buried object sensing considering curved pipeline". In: IEEE Antennas and Wireless Propagation Letters 16 (2017), pp. 2771-2775.

[44] Mritha Ramalingam, Elanchezhian Chinnavan, et al. "A Better Engineering Design: Low Cost Assistance Kit for Manual Wheelchair Users with Enhanced Obstacle Detection." In: Journal of Engineering \& Technological Sciences 47.4 (2015).

[45] Congcong Ma et al. "Posture detection based on smart cushion for wheelchair users". In: Sensors 17.4 (2017), p. 719.

[46] Jaeyong Sung et al. "Unstructured human activity detection from rgbd images". In: 2012 IEEE international conference on robotics and automation. IEEE. 2012, pp. 842-849.

[47] Sameh Neili Boualia and Najoua Essoukri Ben Amara. "Deep Full-Body HPE for Activity Recognition from RGB Frames Only". In: Informatics. Vol. 8. 1. Multidisciplinary Digital Publishing Institute. 2021, p. 2.

[48] Xizhe Yin et al. "Human activity detection based on multiple smart phone sensors and machine learning algorithms". In: 2015 IEEE 19th international conference on computer supported cooperative work in design (CSCWD). IEEE. 2015, pp. 582-587.

[49] Bo Dong and Subir Biswas. "Wearable networked sensing for human mobility and activity analytics: A systems study". In: 2012 Fourth International Conference on Communication Systems and Networks (COMSNETS 2012). IEEE. 2012, pp. 1-6.

[50] J Cho, J Kim, and T Kim. "Smart phone-based human activity classification and energy expenditure generation in building environments". In: Proceedings of the 7th international symposium on sustainable healthy buildings. Vol. 2012. 2012, pp. 97-105.

[51] Matthew Boyle et al. "Poster: Gait-based smartphone user identification". In: Proceedings of the 9th interna- 
tional conference on Mobile systems, applications, and services. 2011, pp. 395-396.

[52] Thomas Plötz, Nils Y Hammerla, and Patrick L Olivier. "Feature learning for activity recognition in ubiquitous computing". In: Twenty-second international joint conference on artificial intelligence. 2011.

[53] Charissa Ann Ronao and Sung-Bae Cho. "Human activity recognition with smartphone sensors using deep learning neural networks". In: Expert systems with applications 59 (2016), pp. 235-244.

[54] L Panini and Rita Cucchiara. "A machine learning approach for human posture detection in domotics applications". In: 12th International Conference on Image Analysis and Processing, 2003. Proceedings. IEEE. 2003, pp. 103-108.

[55] Fei Luo, Stefan Poslad, and Eliane Bodanese. "Human activity detection and coarse localization outdoors using micro-Doppler signatures". In: IEEE Sensors Journal 19.18 (2019), pp. 8079-8094.

[56] Malek Al-Nawashi, Obaida M Al-Hazaimeh, and Mohamad Saraee. "A novel framework for intelligent surveillance system based on abnormal human activity detection in academic environments". In: Neural Computing and Applications 28.1 (2017), pp. 565-572.

[57] Qi Dang et al. "Deep learning based 2d human pose estimation: A survey". In: Tsinghua Science and Technology 24.6 (2019), pp. 663-676.

[58] Yifei Tian et al. "Smart power management Internet of Things system with $5 \mathrm{G}$ and LoRa hybrid wireless networks". In: 5G-Enabled Internet of Things (2019), p. 13.

[59] Yuanzhong Yan and Yongsheng Ou. "Accurate fall detection by nine-axis IMU sensor". In: 2017 IEEE International Conference on Robotics and Biomimetics (ROBIO). IEEE. 2017, pp. 854-859.

[60] Jinkun Han et al. "Lora-based smart IoT application for smart city: an example of human posture detection". In: Wireless Communications and Mobile Computing 2020 (2020).

[61] Audrius Kulikajevas, Rytis Maskeliunas, and Robertas Damaševičius. "Detection of sitting posture using hierarchical image composition and deep learning". In: PeerJ computer science 7 (2021), e442.

[62] Xiaobiao Dai et al. "Multi-task faster R-CNN for nighttime pedestrian detection and distance estimation". In: Infrared Physics \& Technology 115 (2021), p. 103694.

[63] Mandar Gogate, Ahsan Adeel, and Amir Hussain. "Deep learning driven multimodal fusion for automated deception detection". In: 2017 IEEE Symposium Series on Computational Intelligence (SSCI). IEEE. 2017, pp. 1-6.

[64] Mandar Gogate et al. "DNN driven speaker independent audio-visual mask estimation for speech separation". In: arXiv preprint arXiv:1808.00060 (2018).

[65] Kia Dashtipour et al. "A hybrid Persian sentiment analysis framework: Integrating dependency grammar based rules and deep neural networks". In: Neurocomputing 380 (2020), pp. 1-10.
[66] Kia Dashtipour et al. "Exploiting deep learning for Persian sentiment analysis". In: International conference on brain inspired cognitive systems. Springer. 2018, pp. 597-604.

[67] Davide Anguita et al. "A public domain dataset for human activity recognition using smartphones." In: Esann. Vol. 3. 2013, p. 3.

[68] Syed Ikram Shah, Syed Yaseen Shah, and Syed Aziz Shah. "Intrusion Detection through Leaky Wave Cable in Conjunction with Channel State Information". In: 2019 UK/ China Emerging Technologies (UCET). 2019, pp. 1-4. DOI: 10.1109/UCET.2019.8881845.

[69] Eric Wong and Zico Kolter. "Provable defenses against adversarial examples via the convex outer adversarial polytope". In: International Conference on Machine Learning. PMLR. 2018, pp. 5286-5295.

[70] Mohammed Mehedi Hassan et al. "A robust human activity recognition system using smartphone sensors and deep learning". In: Future Generation Computer Systems 81 (2018), pp. 307-313.

[71] Syed Yaseen Shah et al. "A Novel Random Neural Network-based Fall Activity Recognition”. In: 2020 International Conference on UK-China Emerging Technologies (UCET). 2020, pp. 1-4. DOI: 10.1109/ UCET51115.2020.9205414. 